\title{
Detection and miss rates of autofluorescence imaging of adenomatous and polypoid lesions during colonos- copy: a systematic review and meta-analysis
}

Authors

Institutions

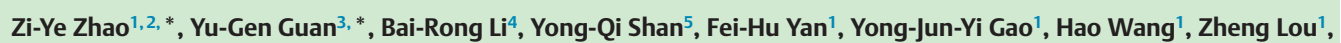
Chuang-Gang Fu' ${ }^{1}$, En-Da Yu ${ }^{1,6}$

Institutions are listed at the end of article. submitted:

13. October 2014

accepted after revision:

31. October 2014

\section{Bibliography}

DOI http://dx.doi.org/

10.1055/s-0034-1391708

Published online: 7.5.2015

Endosc Int Open 2015; 03:

E226-E235

(c) Georg Thieme Verlag KG Stuttgart - New York

E-ISSN 2196-9736

\section{Corresponding author}

\section{En-Da Yu, MD}

Department of Colorectal Surgery

Changhai Hospital

168 Changhai Road

Shanghai

China 200433

Fax: +86-21-31161613

endayu@yeah.net
Background and study aims: Autofluorescence imaging (AFI) is an endoscopic imaging technique used to increase the detection of premalignant gastrointestinal lesions, and it has gradually become popular in recent years. This meta-analysis was performed to examine whether AFI provides greater efficacy in the detection of adenomatous and polypoid lesions and can even prevent the failure to detect a single adenoma or polyp. The aim of the study was to systematically review the efficacy of AFI in increasing detection rates and decreasing miss rates.

Methods: Pertinent articles were identified through a search of databases up to December 2013 that included patients who had undergone two same-day colonoscopies (AFI and white light endoscopy [WLE]), followed by polypectomy. Fixed and random effects models were used to detect significant differences between AFI and WLE in regard to adenoma detection rate (ADR), polyp detection rate (PDR), adenoma miss rate

\section{Introduction}

$\nabla$

The removal of adenomatous lesions by colonoscopy has proved effective in reducing the incidence and disease-associated mortality of colorectal cancer (CRC), with the importance and effectiveness of colonoscopy widely recognized [1, 2]. The research hot spot in colonoscopy is how to improve the efficacy and detection rate, so that lesions are less likely to be missed during examinations. Image-enhanced endoscopy (IEE), serving as a major diagnostic tool in this regard, has been a well-accepted approach within colonoscopy research.

Autofluorescence imaging (AFI), an emerging and promising technique, is able to produce real-time pseudo-color images based on natural tissue

\section{License terms}

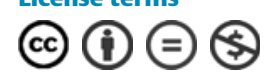

* Dr. Zhao and Dr. Guan contributed equally to this article.
(AMR), polyp miss rate (PMR), and procedural time.

Results: A total of 1199 patients from six eligible studies met the inclusion criteria. No significant differences were found in ADR (odds ratio [OR] 1.01 ; 95\% confidence interval [95\%CI] $0.74-$ 1.37), PDR (OR 0.86; 95\%CI 0.57-1.30), or advanced ADR (OR 1.22; 95\%CI 0.69-2.17). The AMR (OR 0.62; 95\%CI 0.44-0.86) and PMR (OR $0.64 ; 95 \% \mathrm{CI} 0.48-0.85$ ) by AFI were significantly lower than those by WLE. The procedural time of AFI was significantly longer than that of WLE (mean 8.00 minutes; 95\%CI 1.59-14.41). Subgroup meta-analysis for the other characteristics was not performed because of insufficiency of the primary data.

Conclusions: AFI decreases AMR and PMR significantly compared with WLE but does not improve ADR or PDR. AMR and PMR may be decreased by using AFI in flat and small lesions or when less experienced endoscopists perform the procedure.

fluorescence generated from endogenously emitted fluorophores induced by excitation light. The system can visualize benign and malignant lesions based upon differences in tissue fluorescence properties, and thus can reveal early-stage cancers that are less likely detected by conventional white light endoscopy (WLE). Therefore, the clinical application of AFI and research on increasing sensitivity in the detection of premalignant gastrointestinal lesions have markedly increased in recent years. However, because of the limited time of the application of AFI, there is still insufficient evidence regarding whether it can significantly increase adenoma detection rates (ADRs) and polyp detection rates (PDRs) and decrease adenoma miss rates (AMRs) and polyp miss rates (PMRs) in comparison with conventional WLE. Several successful trials have been carried out by researchers all over the world; however, their results are inconsistent and some 
issues require further discussion, so that agreement on this point is yet to be achieved. We performed a systematic review and meta-analysis to find out whether AFI can markedly improve ADRs and PDRs and reduce AMRs and PMRs, and whether there is credible evidence on the value of this technique.

\section{Materials and methods}

$\nabla$

Methods of analysis and inclusion criteria were based on preferred reporting items for systematic reviews and meta-analyses (PRISMA) recommendations ( $\bullet$ Table 1) [3].

\section{Study selection}

A systematic search of the literature up to December 2013 was conducted to include relevant published articles in English (MEDLINE/PubMed, EMBASE, and the Cochrane Library) and in Chinese (SinoMed/CBM). The following terms were used: colonoscopy AND (colonic polyps OR colonic neoplasms OR colonic tumors) AND (autofluorescence imaging OR AFI). Studies published as full articles in English were eligible. Cross-references were also searched manually. The authors of the selected articles were contacted for any missing data.

\section{Inclusion criteria}

Randomized controlled trials (RCTs) in which patients had undergone two same-day colonoscopies (AFI and WLE) were identified, including a tandem design and a back-to-back design. ADR/ PDR or AMR/PMR was set as the primary outcome, while other information about lesions detected was used for subgroup analysis. Indications were screening with average risk or a familial history of CRC, surveillance for colorectal polyps or CRC, and diagnostic colonoscopy.

\section{Exclusion criteria}

Studies aimed to test the diagnostic performance of AFI, and studies that investigated inflammatory bowel diseases were excluded.

\section{Qualitative analysis}

The quality of eligible trials was assessed by using the tool of "risk of bias" according to the Cochrane handbook [4]. Methodologic quality assessment was independently performed by two investigators (Z.-Y. Zhao and Y.-G. Guan). Sequence generation, allocation concealment, blinding (participants/personnel and outcome assessment), data integrity, selective reporting, and other sources of bias were assessed. Based on the method of the trials, "yes" (adequate), "no" (inadequate), or "unclear" was graded individually. Trials with a low risk of bias were the ones fulfilling the adequacy of all the components above, whereas those with a high or medium risk of bias were the ones having one or more of these components regarded as inadequate or unclear. A senior investigator (E.-D. Yu) was consulted in case of any disagreement.

\section{Data extraction}

Data regarding the following variables were extracted from the final selected studies: study design; time and place of the performed studies; single or multiple centers (and whether they were academic centers); total number of patients included; age and sex of the patients; indications and proportion; number of endoscopists and their level of skill; part of the colon investigated; sedation dose; cecum intubation rate; bowel preparation quality; procedural time; number of polyps, adenomas, or advanced adenomas detected within the first and second endoscopies and number of patients with these lesions; number of polyps or adenomas at different locations; and different size categories and different patterns (polypoid or non-polypoid) detected within the first and second endoscopies. The data were extracted, verified, and analyzed by two investigators (Z.-Y. Zhao and Y.-G. Guan) independently. Any difference was resolved by consensus together with a senior investigator (E.-D. Yu).

\section{Outcome measures}

The outcomes measures were (1) ADR and PDR by AFI and WLE for lesions of all types, histology, morphology, location, and size; (2) AMR and PMR by AFI and WLE for lesions of all types, histology, morphology, location, and size; and (3) respective procedural times. The formulas of ADR/PDR and AMR/PMR were as follows. The numerators changed to be numbers of cases with each kind of lesion detected when ADRs/PDRs by histology, morphology, location, and size were calculated. However, the numerators and denominators both changed to be numbers of specific lesions when AMRs/PMRs by histology, morphology, location, and size were calculated.

ADR/PDR = Number of cases with adenomas/polyps detected/Total number of colonoscopies

AMR/PMR (of certain imaging techniques)=Number of cases with adenomas/polyps detected by the other imaging technique/Total number of adenomas/polyps detected by either technique

\section{Statistical analysis}

Relevant extracted data were recorded with Microsoft Excel (Microsoft, Redmond, Washington, USA) and further analyzed with Review Manager Version 5.0.2 (Nordic Cochrane Centre, Copenhagen, Denmark), Stata 12 (Stata Corporation, College Station, Texas, USA) and SPSS Statistics 19 (IBM, Armonk, New York, USA). An $I^{2}$ test was used to assess the heterogeneity of results among the studies. According to the Cochrane Review guidelines, a value of $I^{2}$ above $40 \%$ and a $P$ value below 0.1 were considered as the threshold for heterogeneity. A random effects model was used for the meta-analysis when there was significant heterogeneity among the included studies, and a fixed effects model was used otherwise. Studies displaying statistical heterogeneity were further analyzed for differences in design and results. The outcomes were expressed as odds ratio (OR) and $95 \%$ confidence interval $(95 \% \mathrm{CI})$ or mean $(95 \% \mathrm{CI}), I^{2}$, and $P$ value for heterogeneity with forest plots. Publication bias was assessed with the BeggMazumdar Kendall's test. Sensitivity analysis was also performed by comparing the results of the fix effects model and the random effects model.

\section{Results \\ $\nabla$}

A total of 143 studies were retrieved (138 in English and 5 in Chinese), of which 129 were excluded for nonrandomized design (125 in English and 4 in Chinese). Another 2 studies, of patients with inflammatory bowel diseases, were excluded $[5,6]$, and 3 studies were not included because they evaluated the diagnostic performance of AFI (sensitivity, specificity, or negative predictive value) [7-9]. Another 3 trials were excluded because of inappropriate interventions (intravenous injection of antibody or AFI plus transparent cap) [10 - 12]. Finally, 6 RCTs were included (5 in English and 1 in Chinese) [13-18], which were all published as 
Table 1 Preferred reporting items for systematic reviews and meta-analyses (PRISMA) 2009 checklist.

\begin{tabular}{|c|c|c|c|}
\hline Section/topic & Item & Checklist item & $\begin{array}{l}\text { Reported on } \\
\text { page number }\end{array}$ \\
\hline \multicolumn{4}{|l|}{ Title } \\
\hline Title & 1 & Identify the report as a systematic review, meta-analysis, or both. & 1 \\
\hline \multicolumn{4}{|l|}{ Abstract } \\
\hline Structured summary & 2 & $\begin{array}{l}\text { Provide a structured summary including, as applicable, background; objectives; data sources; } \\
\text { study eligibility criteria, participants, and interventions; study appraisal and synthesis meth- } \\
\text { ods; results; limitations; conclusions and implications of key findings; systematic review re- } \\
\text { gistration number. }\end{array}$ & $1-2$ \\
\hline \multicolumn{4}{|l|}{ Introduction } \\
\hline Rationale & 3 & Describe the rationale for the review in the context of what is already known. & 3 \\
\hline Objectives & 4 & $\begin{array}{l}\text { Provide an explicit statement of questions being addressed with reference to participants, } \\
\text { interventions, comparisons, outcomes, and study design (PICOS). }\end{array}$ & 3 \\
\hline \multicolumn{4}{|l|}{ Methods } \\
\hline Protocol and registration & 5 & $\begin{array}{l}\text { Indicate if a review protocol exists, if and where it can be accessed (e. g., Web address), and, if } \\
\text { available, provide registration information including registration number. }\end{array}$ & 4 \\
\hline Eligibility criteria & 6 & $\begin{array}{l}\text { Specify study characteristics (e. g., PICOS, length of follow-up) and report characteristics } \\
\text { (e. g., years considered, language, publication status) used as criteria for eligibility, giving } \\
\text { rationale. }\end{array}$ & 4 \\
\hline Information sources & 7 & $\begin{array}{l}\text { Describe all information sources (e. g., databases with dates of coverage, contact with study } \\
\text { authors to identify additional studies) in the search and date last searched. }\end{array}$ & 4 \\
\hline Search & 8 & $\begin{array}{l}\text { Present full electronic search strategy for at least one database, including any limits used, such } \\
\text { that it could be repeated. }\end{array}$ & 4 \\
\hline Study selection & 9 & $\begin{array}{l}\text { State the process for selecting studies (i. e., screening, eligibility, included in systematic } \\
\text { review, and, if applicable, included in the meta-analysis). }\end{array}$ & 4 \\
\hline Data collection process & 10 & $\begin{array}{l}\text { Describe method of data extraction from reports (e. g., piloted forms, independently, in } \\
\text { duplicate) and any processes for obtaining and confirming data from investigators. }\end{array}$ & $5-6$ \\
\hline Data items & 11 & $\begin{array}{l}\text { List and define all variables for which data were sought (e. g., PICOS, funding sources) and any } \\
\text { assumptions and simplifications made. }\end{array}$ & 5 \\
\hline $\begin{array}{l}\text { Risk of bias in individual } \\
\text { studies }\end{array}$ & 12 & $\begin{array}{l}\text { Describe methods used for assessing risk of bias of individual studies (including specification } \\
\text { of whether this was done at the study or outcome level), and how this information is to be used } \\
\text { in any data synthesis. }\end{array}$ & 4 \\
\hline Summary measures & 13 & State the principal summary measures (e. g., risk ratio, difference in means). & $5-6$ \\
\hline Synthesis of results & 14 & $\begin{array}{l}\text { Describe the methods of handling data and combining results of studies, if done, including } \\
\text { measures of consistency }\left(\text { e. g., } I^{2}\right) \text { for each meta-analysis. }\end{array}$ & $5-6$ \\
\hline Risk of bias across studies & 15 & $\begin{array}{l}\text { Specify any assessment of risk of bias that may affect the cumulative evidence (e. g., publica- } \\
\text { tion bias, selective reporting within studies). }\end{array}$ & 4 \\
\hline Additional analyses & 16 & $\begin{array}{l}\text { Describe methods of additional analyses (e. g., sensitivity or subgroup analyses, meta- } \\
\text { regression), if done, indicating which were pre-specified. }\end{array}$ & $5-6$ \\
\hline \multicolumn{4}{|l|}{ Results } \\
\hline Study selection & 17 & $\begin{array}{l}\text { Give numbers of studies screened, assessed for eligibility, and included in the review, with } \\
\text { reasons for exclusions at each stage, ideally with a flow diagram. }\end{array}$ & 7 \\
\hline Study characteristics & 18 & $\begin{array}{l}\text { For each study, present characteristics for which data were extracted (e. g., study size, PICOS, } \\
\text { follow-up period) and provide the citations. }\end{array}$ & $7,22-23$ \\
\hline Risk of bias within studies & 19 & $\begin{array}{l}\text { Present data on risk of bias of each study and, if available, any outcome level assessment (see } \\
\text { item 12). }\end{array}$ & \\
\hline $\begin{array}{l}\text { Results of individual stud- } \\
\text { ies }\end{array}$ & 20 & $\begin{array}{l}\text { For all outcomes considered (benefits or harms), present for each study: (a) simple summary } \\
\text { data for each intervention group and (b) effect estimates and confidence intervals, ideally with } \\
\text { a forest plot. }\end{array}$ & $7-11$ \\
\hline Synthesis of results & 21 & $\begin{array}{l}\text { Present the main results of the review. If meta-analyses are done, include confidence intervals } \\
\text { and measures of consistency for each. }\end{array}$ & $7-11$ \\
\hline Risk of bias across studies & 22 & Present results of any assessment of risk of bias across studies (see item 15). & 7 \\
\hline Additional analysis & 23 & $\begin{array}{l}\text { Give results of additional analyses, if done (e. g., sensitivity or subgroup analyses, meta- } \\
\text { regression [see item [16])]. }\end{array}$ & 11 \\
\hline \multicolumn{4}{|l|}{ Discussion } \\
\hline Summary of evidence & 24 & $\begin{array}{l}\text { Summarize the main findings, including the strength of evidence for each main outcome; } \\
\text { consider their relevance to key groups (e. g., health care providers, users, and policy makers). }\end{array}$ & 12 \\
\hline Limitations & 25 & $\begin{array}{l}\text { Discuss limitations at study and outcome level (e. g., risk of bias) and at review level (e. g., } \\
\text { incomplete retrieval of identified research, reporting bias). }\end{array}$ & 12 \\
\hline Conclusions & 26 & $\begin{array}{l}\text { Provide a general interpretation of the results in the context of other evidence, and implica- } \\
\text { tions for future research. }\end{array}$ & $12-15$ \\
\hline \multicolumn{4}{|c|}{ (1) } \\
\hline Funding & 27 & $\begin{array}{l}\text { Describe sources of funding for the systematic review and other support (e. g., supply of data), } \\
\text { role of funders for the systematic review. }\end{array}$ & 16 \\
\hline
\end{tabular}




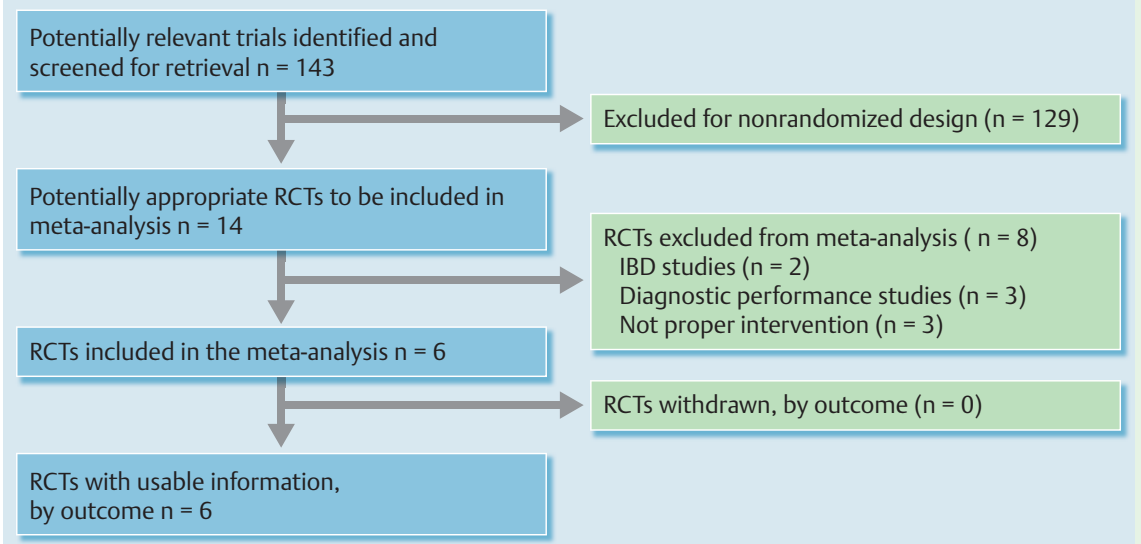

Fig. 1 Quality of reporting of meta-analyses (QUORUM) flow diagram of included and excluded studies. RCT, randomized controlled trial; IBD, inflammatory bowel disease. full articles ( $\bullet$ Fig. 1). Quality assessment was performed for each selected trial $(\bullet$ Fig. 2$)$.

\section{Characteristics of the included studies}

General characteristics of the included articles are presented in - Table 2.

All studies applied the following exclusion criteria: history of inflammatory bowel disease, history of surgical resection, lack of informed consent, poor bowel preparation $(>10 \%$ of mucosal area obscured), and poor medical condition of the patient that could not allow two consecutive colonoscopies. Five studies [14-18] reported inability to reach the cecum that was regarded as an exclusion criterion.

The main indications were a family history of CRC and surveillance of colorectal polyps ( $\bullet$ Table 2 ). The proportion of patients screened in the different studies varied from $0 \%$ in three studies to $43.2 \%$ in the study by Moriichi et al. Because the prevalence of colorectal polyps in the patients screened was relatively low, they may not have been the optimal population for a study of miss rates. Based on the indications for colonoscopy and the mean total number of polyps per patient (MTPD; i. e., the total number of polyps detected divided by the total number of people undergoing colonoscopy), the patients in three [15 - 17], two [13,14], and one [18] of the studies were defined as high, medium, and low risk populations for polyps, respectively.

A randomized tandem design comparing the AMR and PMR of AFI and WLE was applied in the five studies included. Colonoscopic techniques evaluated included the following: standard resolution endoscopy versus high resolution endoscopy (HRE) plus AFI [16], and WLE versus AFI $[14,15,17,18]$. A study by Moriichi et al. adopted another design, called back-to-back colonoscopies, in which the two endoscopists were not aware of each other's observation. In the tandem design, ADR and PDR were extracted from the former examination, and AMR and PMR were determined by the difference of the two examinations; in the backto-back design, on the other hand, ADR/PDR was determined by the respective examinations of different techniques, and AMR/ PMR was worked out through the difference between the total number of lesions and the number detected by the respective techniques. We combined the data from all trials, and the influence of the different designs was taken into account within discussion.

We observed differences between the prevalence of polyps in the study populations. The MTPD ranged from 0.45 to 2.08 ( $\bullet$ Table 2 ). In the study by Jiang et al., participants with an average risk of CRC were largely involved, explaining the low MTPD. In the stud-

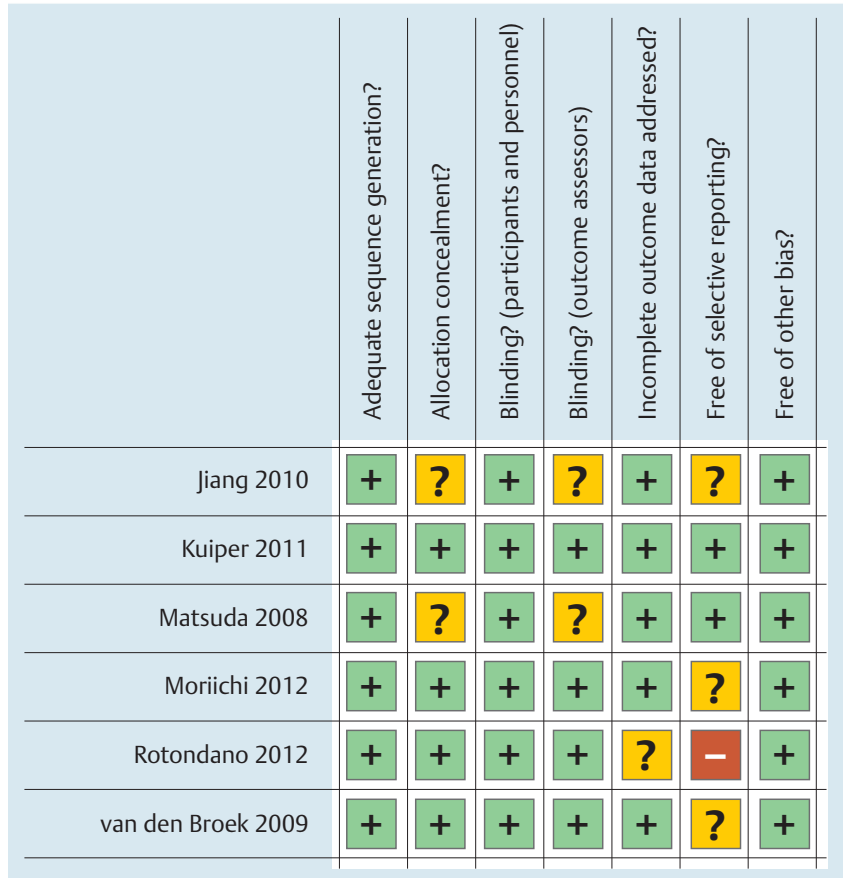

Fig.2 Quality summary: review authors' judgments about each methodologic quality item for each included study.

ies by Matsuda et al. and Moriichi et al., the colonoscopic examinations were limited to the proximal colon and to the sigmoid colon and rectum, respectively, which largely explains the low MTPD. The study by Kuiper et al. showed that PDR indicated the distribution of the lesions within the population. However, the other two studies failed to elucidate whether the high MTPD was attributable to a small number of patients with many polyps or whether the polyps were evenly distributed among patients owing to no available PDR provided.

\section{Adenoma detection rate of all types of lesions}

Five studies showed the data of cases with adenomas detected used for ADR analysis ( $\bullet$ Fig. 3). The pooled data (fixed effects model), regardless of size and location, showed no significant difference by means of AFI or conventional WLE (OR 1.01; $95 \% \mathrm{CI}$ $0.74-1.37 ; P=0.96)$. There was no significant heterogeneity among the studies $\left(P=0.67 ; I^{2}=0\right)$. 


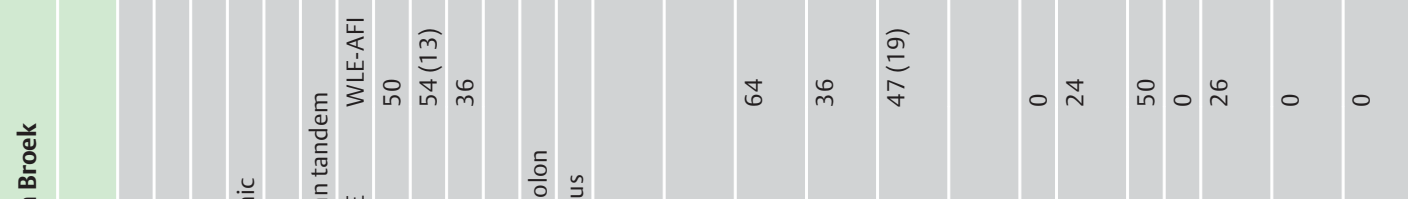

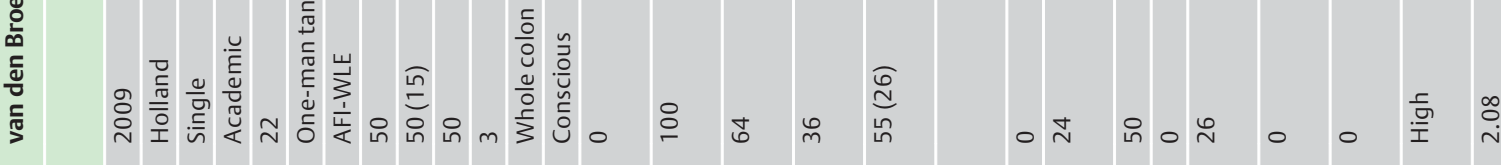

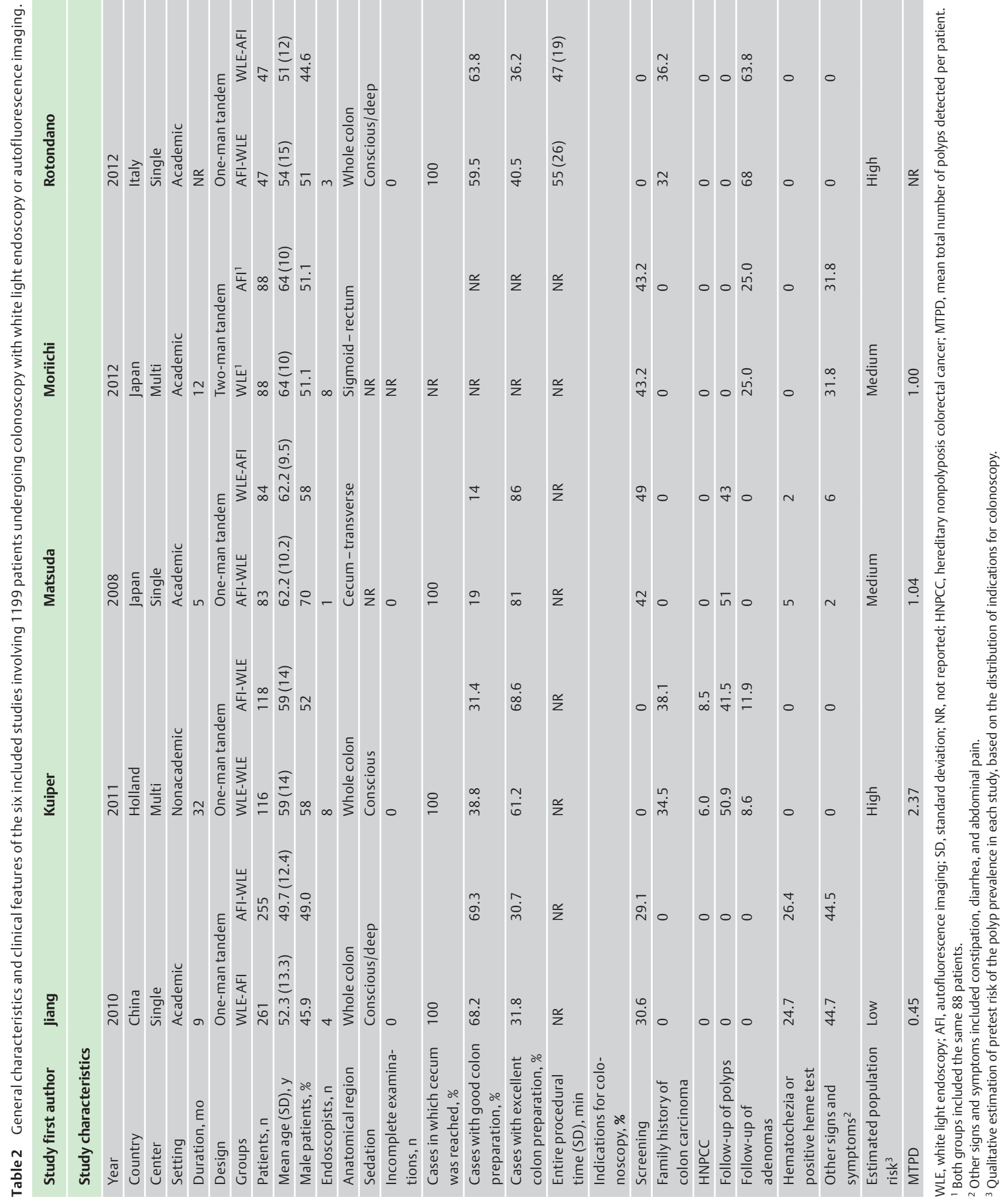




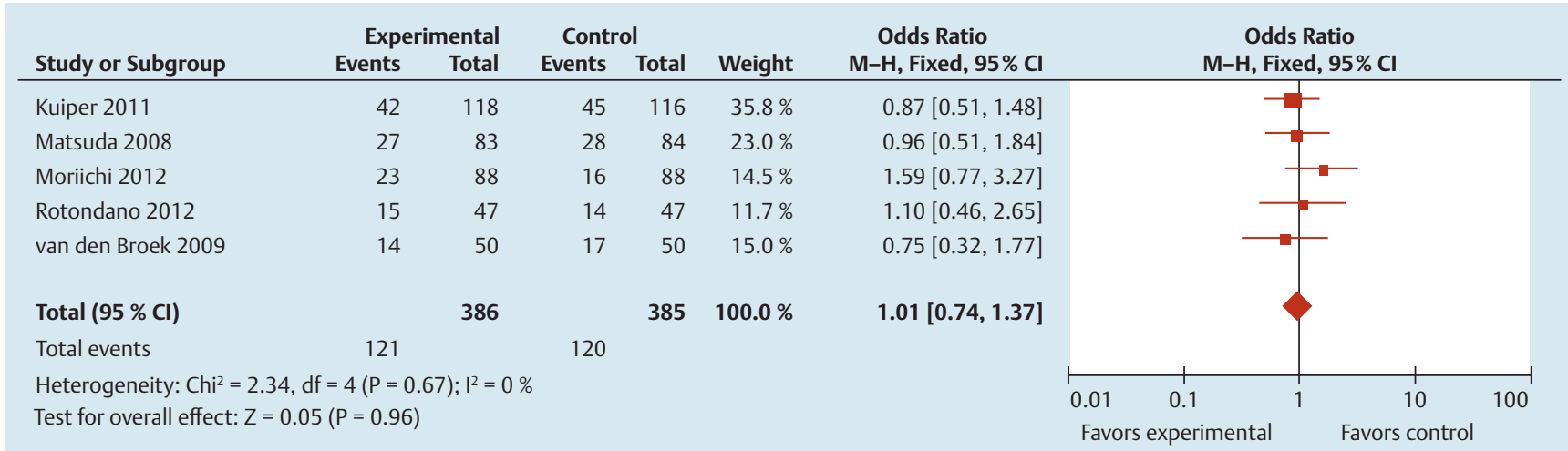

Fig. 3 Comparison of adenoma detection rates of autofluorescence imaging and white light endoscopy. M-H, Mantel- Haenszel; $\mathrm{Cl}$, confidence interval.

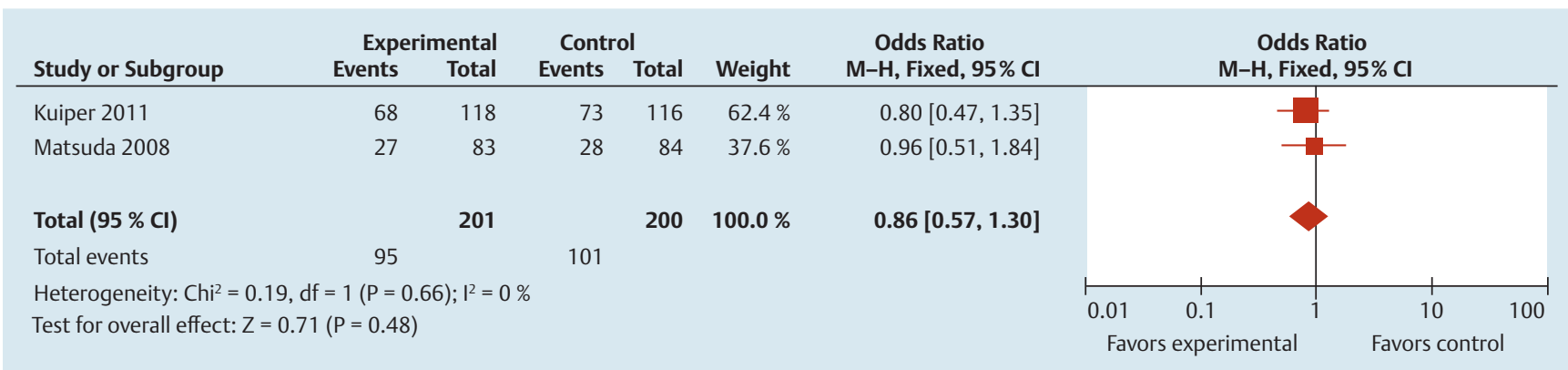

Fig. 4 Comparison of advanced adenoma detection rates of autofluorescence imaging and white light endoscopy. M-H, Mantel-Haenszel; $\mathrm{Cl}$, confidence interval.

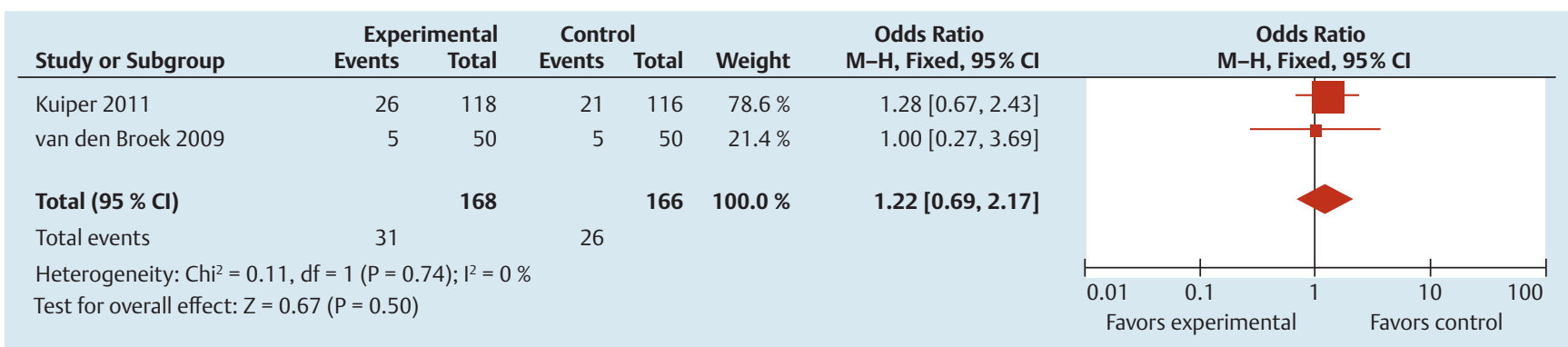

Fig. 5 Comparison of polyp detection rates of autofluorescence imaging and white light endoscopy. M-H, Mantel- Haenszel; Cl, confidence interval.

\section{Polyp detection rate of all types of lesions}

The numbers of cases of polyps detected were shown in only two studies $[14,16]$. Although the data used for PDR analysis were insufficient, they were still considered for analysis in this study ( $\bullet$ Fig. 4). The pooled data, regardless of size and location, showed no significant difference between AFI and WLE (OR $0.86 ; 95 \% \mathrm{Cl} 0.57-1.30 ; P=0.71$ ), with no obvious heterogeneity $\left(P=0.66 ; I^{2}=0\right)$.

\section{Adenoma detection rate by histology, morphology,} location, and size of lesions

Two studies reported the analysis of advanced adenomas ( $\bullet$ Fig. 5) $[15,16]$. The pooled data for advanced ADR (fix effects analysis) showed no significant difference (OR 1.22; $95 \% \mathrm{CI}$ $0.69-2.17 ; P=0.50)$. There was no significant heterogeneity $(P$ $=0.74 ; I^{2}=0$ ).
As for the morphology of adenomas, the ADR of polypoid and non-polypoid types was extracted from only one study [13]. Some other studies also took morphology into consideration, but they were not qualified for analysis because of insufficient cases with individual types. The only available data showed no significant difference between the two colonoscopic techniques in either the polypoid $(P=0.680)$ or non-polypoid type $(P=$ $0.106)$.

There were no data for location and size analysis because none of the studies provided information about the number of cases with the specific types of adenomas.

\section{Polyp detection rate by morphology, location,} and size of lesions

Similar to the situation for specific ADR analysis, there was insufficient information for specific PDR analysis. 


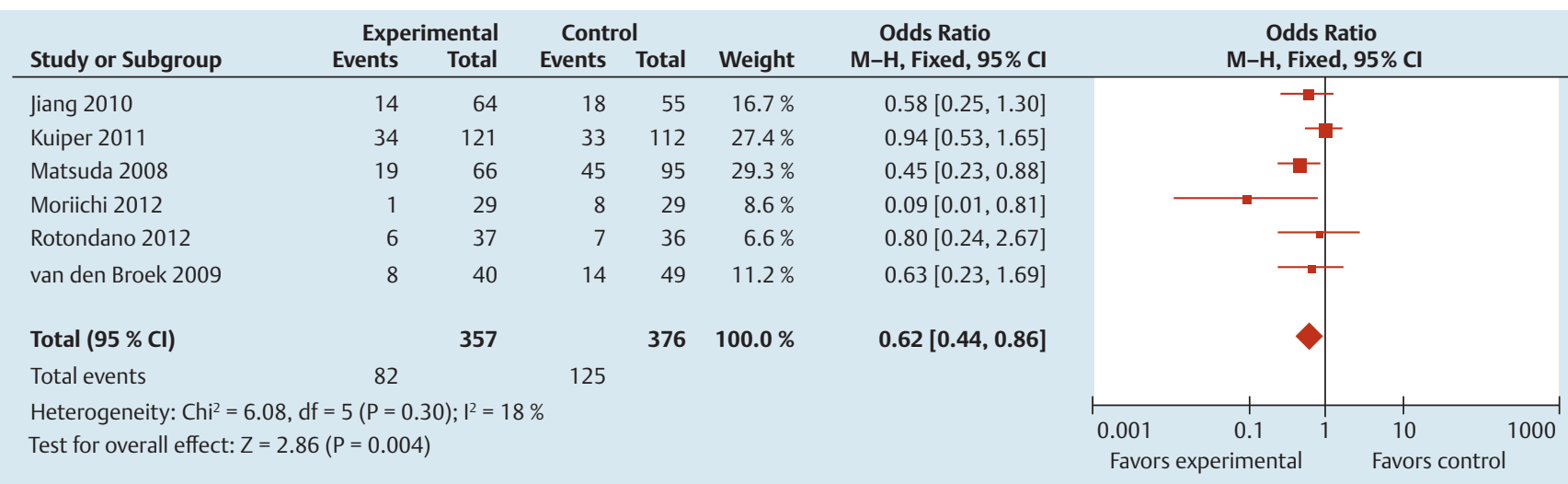

Fig. 6 Comparison of adenoma miss rates of autofluorescence imaging and white light endoscopy. $\mathrm{M}-\mathrm{H}$, Mantel- $\mathrm{Haenszel}$; $\mathrm{Cl}$, confidence interval.

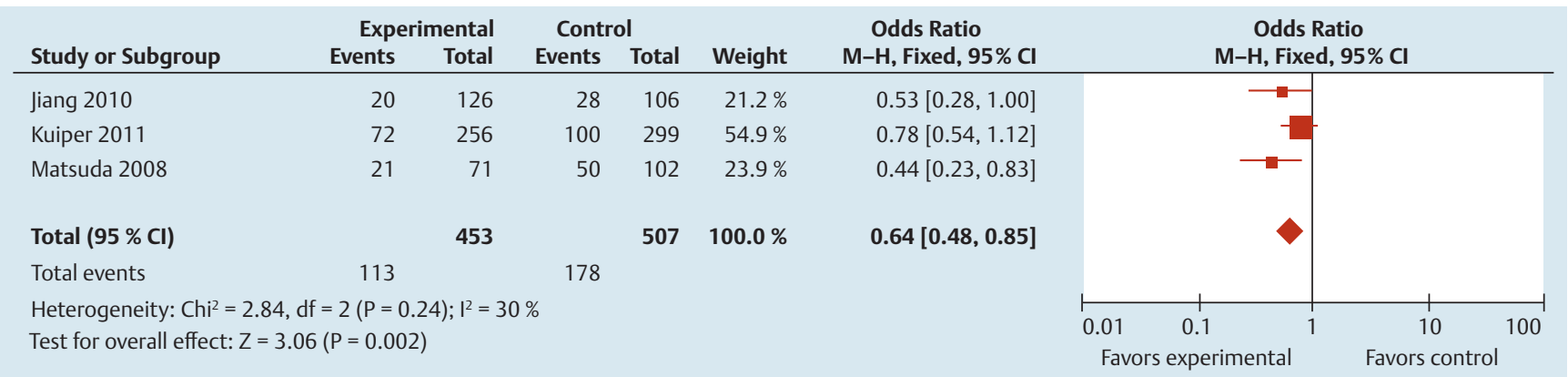

Fig.7 Comparison of polyp miss rates of autofluorescence imaging and white light endoscopy. M-H, Mantel- Haenszel; Cl, confidence interval.

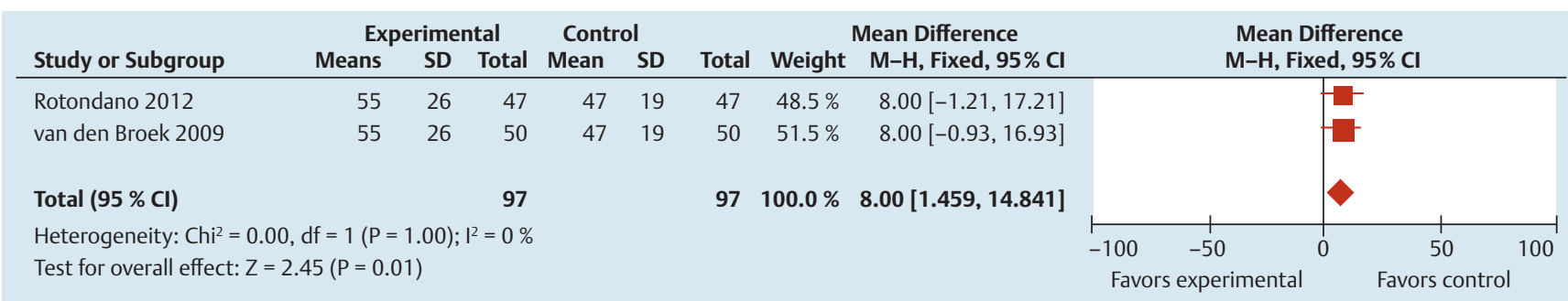

Fig. 8 Comparison of procedural times of autofluorescence imaging and white light endoscopy for whole colon. SD, standard deviation; IV, inverse variance; $\mathrm{Cl}$, confidence interval.

\section{Adenoma miss rate of all types of lesions}

The meta-analysis of AMRs is shown in Fig. 6. The six studies reported a total of 704 adenomas of all types and all sizes. Because the back-to-back design doubled the number of participants in the study by Moriichi et al., these data (29 adenomas) were counted twice when the total number of participants and lesions was calculated. Therefore, the total number of adenomas was 733, of which 207 adenomas were discovered at the second colonoscopy alone. The pooled data showed that the AMR of AFI was significantly lower than that of WLE (OR 0.62; 95\%CI $0.44-$ $0.86 ; P=0.004)$. There was no significant heterogeneity $(P=0.30$; $\left.I^{2}=18 \%\right)$.

\section{Polyp miss rate of all types of lesions}

Four studies reported a total of 1048 polyps of all types and all sizes. When a fixed effects model was used, the result showed significant heterogeneity $\left(P=0.0002 ; I^{2}=85 \%\right)$. Therefore, we applied the random effects analysis, but the heterogeneity was not well decreased. We attributed the high degree of heterogeneity to the data from the study of Moriichi et al. because of its unique study design. After this study had been excluded, there was no significant heterogeneity among the remaining studies $(P=0.24$; $I^{2}=30 \%$ ) ( Fig. 7). The pooled data showed that the PMR yielded by AFI was significantly lower than that of conventional WLE (OR $0.64 ; 95 \%$ CI $0.48-0.85 ; P=0.002$ ).

Adenoma miss rate by histology, morphology, location, and size of lesions

Two studies reported the analysis of advanced adenomas [15, 16], but neither group in the study by van den Broek et al. had advanced adenomas missed, which meant that the data were not suitable to be brought into the meta-analysis. Neither of the two studies showed a significant difference between AFI and WLE (Kuiper et al.: $P=0.283$; van den Broek et al.: $P=1.00$ ).

Although all the studies except the one by Moriichi et al. had analysis for miss rate by morphology, it was still disqualified from 
Table 3 Sensitivity analysis.

\begin{tabular}{lllllll} 
& ADR & Advanced ADR & PDR & AMR & PMR & \multicolumn{1}{c}{$\begin{array}{l}\text { Procedural time, } \\
\text { min }\end{array}$} \\
\hline $\begin{array}{l}\text { Fixed effect } \\
\text { model OR } \\
(95 \% \mathrm{Cl})\end{array}$ & $1.01(0.74-1.37)$ & $1.22(0.69-2.17)$ & $0.68(0.57-1.30)$ & $0.62(0.44-0.86)$ & $0.64(0.48-0.85)$ & $8.00(1.59-14.41)$ \\
\hline $\begin{array}{l}\text { Random effect } \\
\text { model OR } \\
(95 \% \mathrm{Cl})\end{array}$ & $1.01(0.74-1.37)$ & $1.22(0.68-2.17)$ & $0.86(0.57-1.30)$ & $0.62(0.42-0.92)$ & $0.61(0.43-0.88)$ & $8.00(1.59-14.41)$ \\
\hline
\end{tabular}

ADR, adenoma detection rate; PDR, polyp detection rate; AMR, adenoma miss rate; PMR, polyp miss rate; OR, odds ratio; $\mathrm{Cl}$, confidence interval.

analysis because of the heterogeneity of the relevant data. Two studies reported no significant difference in the AMRs for both morphology types between AFI and WLE, but no specific data were shown $[15,16]$. Matsuda et al. put the number of cases detected by one kind of technique and missed cases by the other kind together for analysis; thus, no precise data were available for our meta-analysis. The data of the other two studies cannot be combined either because one set of data was about adenomas only [17], while the other was about all kinds of polypoid lesions [18]. The result of the only available data by Rotondano et al. showed no significant difference in the AMRs for both types of morphology between AFI and WLE (polypoid lesions, $P=0.745$; non-polypoid lesions, $P=1.00$ ). The situation was the same for the analysis of location and size.

\section{Polyp miss rate by morphology, location,} and size of lesions

The study by Jiang et al. was the only one that analyzed PMR in regard to different characteristics. The PMR of AFI was significantly lower than that of WLE for the non-polypoid type ( $P=$ 0.003 ; OR 0.26 ; $95 \%$ CI $0.10-0.64)$, but the difference was not significant for the polypoid type $(P=0.691)$.

As for the location of lesions, there was no significant difference in PMR in the proximal part $(P=0.305)$ and distal part $(P=0.127)$. The most commonly reported polyp sizes were 1 to $5 \mathrm{~mm}, 6$ to 9 $\mathrm{mm}$, and $10 \mathrm{~mm}$ or larger, which were available in this study. The data for small polyps $(1-5 \mathrm{~mm})$ showed a significant difference (PMR of AFI was lower) ( $P=0.013$; OR 0.34 ; 95\%CI $0.14-0.81$ ). The data for medium-size $(6-9 \mathrm{~mm})$ and large polyps $(10 \mathrm{~mm}$ and larger) showed no significant difference (medium, $P=0.823$; large, $P=0.500)$.

\section{Procedural time}

Two studies reported the procedural time for the entire colon ( $\bullet$ Fig. 8). The pooled data indicated that the procedural time of AFI was significantly longer than that of WLE (mean 8.00 minutes; $95 \%$ CI $1.59-14.41 ; P=0.01)$, with no significant heterogeneity $\left(P=1.00 ; I^{2}=0\right)$.

\section{Publication bias}

Tests for funnel plot asymmetry were used only for meta-analysis with at least 10 studies included; otherwise, the power of the tests was too low to distinguish chance from real asymmetry [4]. Therefore, publication bias was assessed with the Begg-Mazumdar Kendall's test for ADR, AMR, and PMR, of which there were three or more studies involved in the meta-analysis. The result indicated that no bias was found for ADR $(t=0.39 ; P=0.723)$, $\operatorname{AMR}(t=-1.59 ; P=0.186)$, or $\mathrm{PMR}(t=-3.97 ; P=0.157)$.

\section{Sensitivity analysis}

Sensitivity analysis was performed to examine the robustness of the result by changing the model. The result indicated that all results were robust ( $\bullet$ Table 3 ).

\section{Discussion \\ $\nabla$}

In this systematic review, we analyzed the ADR/PDR and AMR/ PMR of AFI and WLE according to the histology, morphology, location, and size of lesions. The available evidence, however, was based on a relatively small number of heterogeneous studies that included a total of 1199 cases. Our results indicated that AFI significantly decreased the AMR/PMR when compared with conventional WLE but did not markedly increase the ADR/PDR. We conclude that colorectal polyps or adenomas would less likely be missed with AFI, but the number of cases with adenomas or polyps may not be different. AFI did reduce the number of missed lesions, but the ADR and PDR may not be influenced because they were calculated for patients who have lesions. Adding one lesion detected for a patient who already has lesions detected does not change the ADR or PDR. Therefore, we can see that the miss rate is more sensitive than the detection rate. This work provides more comprehensive evidence and is a supplement to the area of IEE research because meta-analyses have been conducted for chromoendoscopy [19] and narrow-band imaging (NBI) [20-24].

According to the existing literature, the PMR significantly increases at a rate that is inversely related to the size of the polyp [25]; moreover, there is new evidence indicating that AFI can decrease the PMR of small polyps, although with limited number of cases, and the article was written in Chinese. Nevertheless, there is still hope that AFI may help to decrease the miss rate of small polyps and adenomas significantly, based on future RCTs with larger sample sizes.

It is not certain whether autofluorescence can decrease the PMR of medium-size polyps. A systematic review reported a poor PMR of $13 \%$ for medium-size polyps, which may be a serious issue in clinical practice [25]. Therefore, the recommendation to report medium-size polyps requires more attention [26]. The ability of AFI in this aspect requires further exploration.

The limited evidence indicated that the PMRs for non-polypoid lesions (flat lesions) by AFI and WLE differed from each other. Some other studies have given similar results. The result of the study of Takeuchi et al., which focused on the efficacy of AFI with a transparent hood, suggested that AFI colonoscopy with a transparent detected significantly more colorectal neoplasms than did WLE without a transparent hood [11]. In the subgroup analysis of the polypoid/flat neoplasms detected, mounting a transparent hood helped to detect more polypoid neoplasms, 
and AFI observation detected more flat neoplasms. Current opinion regarding this subject is that AFI may be more adequate than WLE to detect flat lesions, but its ability is affected easily by the area of observation. While a transparent hood can help to detect lesions behind the colonic folds by pushing them, mounting a transparent hood and AFI observation might complement each other, and the combination would be effective for the detection of both polypoid and flat neoplasms [10]. Future evidence of good quality is needed to prove this viewpoint and make AFI with a transparent hood a standard tool for CRC surveillance.

As for methodology, the design of tandem colonoscopy has been regarded as the gold standard for miss rate research for a long time. A tandem design includes two same-day colonoscopies within one patient, both of them with polypectomy. In such studies, the AMR/PMR is expressed as the number of polyps/adenomas detected only during the second colonoscopy relative to the number found during both examinations. However, there is yet another design available as an alternative option, which is a back-to-back design (used in the study of Moriichi et al.). A back-to-back design also includes two same-day colonoscopies for each patient, but they are performed by two different endoscopists, and polypectomy is performed only during the second examination. The first advantage of this design is that it makes a blind trial possible, in that the two independent investigators are unaware of each other's observation and the sequencing is insignificant. One examination can be regarded as the second examination to the other one, and the extra lesions detected by one technique are regarded as lesions missed by the other. With this method, the sample size will be doubled, which is the second advantage; moreover, because two individual investigators are engaged in the examinations of one patient, it is possible to perform analysis with endoscopists of different levels of experience. As the result of the study of Moriichi et al. showed, AFI dramatically increased the ADR (30.3\%) in comparison with HRE $(7.7 \%$; $P<$ 0.05 ) in endoscopists with less experience. Conversely, the ADR of HRE in experienced endoscopists $(22.6 \%)$ was not significantly different from that of AFI (23.6\%). However, the differences between endoscopists may become a confounding factor when other analyses of AMR/PMR are performed. This may also happen in tandem studies when the two examinations are performed by two different endoscopists. Therefore, tandem colonoscopies performed by a single investigator are a good way to reduce bias caused by observers.

As for meta-analysis, including studies of different designs will produce heterogeneity, which can decrease the power of evidence, just like the analysis of the PMR of all types of lesions. This time, we must exclude the study that used a back-to-back design to make the analysis more acceptable.

The quality of studies included is basically acceptable, but there are still drawbacks within most of them. Lack of necessary and precise information is one common problem. After the authors had reported the primary outcome, their attitude about reporting other research data became variable. Some authors gave very precise information about polyps or adenomas detected and missed, and the number of cases used for calculating the detection rate. Of these six studies, the one by Kuiper et al. had the best quality and the most precise data. This helped us make a more comprehensive subgroup analysis and find more latent problems. Nevertheless, other studies failed to report a more comprehensive coverage of data.

Another problem that makes subgroup meta-analysis difficult is the authors' variable ways of processing their data. As mentioned before, there are accepted classifications for size and location. However, investigators sometimes change the classification as needed. Other than that, combining different parts of the data for comparison instead of presenting the original data makes the data unsuitable for meta-analysis $[14,18]$.

Based on the preceding discussion, there are clues indicating that the AMR/PMR may be decreased by using AFI in flat and small lesions or when procedures are performed by less experienced endoscopists, which will further the use of AFI in clinical practice. The analysis for the diagnostic performance of AFI was not included in this study. A newly published meta-analysis has provided a comprehensive analysis for this aspect of several IEE techniques, including AFI. The results indicated that all endoscopic imaging techniques other than AFI could be used by welltrained endoscopists to achieve a more reliable optical diagnosis of colonic lesions in daily practice, which was not beneficial for AFI, but AFI still has its advantage of detecting lesions that can secure its place in colonoscopic examinations. Combining IEE techniques may be the best way to achieve maximum effectiveness, and some manufacturers have already developed new devices for combining IEE techniques. Endoscopic trimodal imaging (ETMI) with the combination of WLE, NBI, and AFI is a good example $[6,27]$. In fact, in the proper combination, AFI with other IEE techniques will make colonoscopy more effective, more convenient, and less labor-consuming.

Competing interests: None. This research was supported by grants of Discipline Constructions Project 1255 of Changhai Hospital (CH125530800).

\section{Institutions}

${ }^{1}$ Department of Colorectal Surgery, Changhai Hospital, Second Military Medical University, Shanghai, China

2 Department of Surgery, No. 273 Hospital of People's Liberation Army, Korla, Xinjiang Autonomous Region, China

${ }^{3}$ Medical Team, Unit 66013 of People's Liberation Army, Shijiazhuang, Hebei Province, China

${ }^{4}$ Department of Gastroenterology, Air Force General Hospital of People's Liberation Army, Beijing, China

${ }^{5}$ Department of General Surgery, Shengyang General Hospital of People's Liberation Army, Shenyang, Liaoning Province, China

${ }^{6}$ Center of Digestive Endoscopy, Changhai Hospital, Second Military Medical University, Shanghai, China

\section{References}

1 Winawer SJ, Zauber AG, Ho MN et al. Prevention of colorectal cancer by colonoscopic polypectomy. The National Polyp Study Workgroup. N Engl J Med 1993; 329: 1977-1981

2 Zauber AG, Winawer SJ, O'Brien MJ et al. Colonoscopic polypectomy and long-term prevention of colorectal-cancer deaths. N Engl J Med 2012; 366: 687-696

3 Moher D, Liberati A, Tetzlaff J et al. Preferred reporting items for systematic reviews and meta-analyses: the PRISMA statement. Ann Intern Med 2009; 151: 264-269, W64

4 Higgins JP, Green $S$ eds. Cochrane handbook for systematic reviews of interventions version 5.0.2 [updated September 2009]. Chichester, UK: John Wiley \& Sons; 2009

5 Matsumoto T, Nakamura S, Moriyama T et al. Autofluorescence imaging colonoscopy for the detection of dysplastic lesions in ulcerative colitis: a pilot study. Colorectal Dis 2010; 12: e291 - e297

6 van den Broek FJ, Fockens P, van Eeden $S$ et al. Endoscopic tri-modal imaging for surveillance in ulcerative colitis: randomised comparison of high-resolution endoscopy and autofluorescence imaging for neoplasia detection; and evaluation of narrow-band imaging for classification of lesions. Gut 2008; 57: 1083-1089

7 Ignjatovic A, East JE, Guenther T et al. What is the most reliable imaging modality for small colonic polyp characterization? Study of white- 
light, autofluorescence, and narrow-band imaging. . Endoscopy 2011; 43: $94-99$

8 Sato R, Fujiya $M$, Watari $J$ et al. The diagnostic accuracy of high-resolution endoscopy, autofluorescence imaging and narrow-band imaging for differentially diagnosing colon adenoma. Endoscopy 2011; 43: $862-868$

9 Moriichi K, Fujiya M, Sato $R$ et al. Autofluorescence imaging and the quantitative intensity of fluorescence for evaluating the dysplastic grade of colonic neoplasms. Int J Colorectal Dis 2012; 27: 325-330

10 Takeuchi $Y$, Inoue T, Hanaoka $N$ et al. Surveillance colonoscopy using a transparent hood and image-enhanced endoscopy. Dig Endosc 2010; 22: S47-S53

11 Takeuchi $Y$, Inoue T, Hanaoka $N$ et al. Autofluorescence imaging with a transparent hood for detection of colorectal neoplasms: a prospective, randomized trial. Gastrointest Endosc 2010; 72: 1006 - 1013

12 Folli S, Wagnieres G, Pelegrin A et al. Immunophotodiagnosis of colon carcinomas in patients injected with fluoresceinated chimeric antibodies against carcinoembryonic antigen. Proc Natl Acad Sci U S A 1992; 89: 7973 - 7977

13 Moriichi K, Fujiya M, Sato $R$ et al. Back-to-back comparison of autofluorescence imaging (AFI) versus high resolution white light colonoscopy for adenoma detection. BMC Gastroenterol 2012; $12: 75$

14 Matsuda T, Saito Y, Fu KI et al. Does autofluorescence imaging videoendoscopy system improve the colonoscopic polyp detection rate? - a pilot study. Am J Gastroenterol 2008; 103: 1926-1932

15 van den Broek FJ, Fockens P, Van Eeden S et al. Clinical evaluation of endoscopic trimodal imaging for the detection and differentiation of colonic polyps. Clin Gastroenterol Hepatol 2009; 7: 288 - 295

16 Kuiper T, van den Broek FJ, Naber AH et al. Endoscopic trimodal imaging detects colonic neoplasia as well as standard video endoscopy. Gastroenterology 2011; 140: 1887-1894

17 Rotondano G, Bianco MA, Sansone S et al. Trimodal endoscopic imaging for the detection and differentiation of colorectal adenomas: a prospective single-centre clinical evaluation. Int J Colorectal Dis 2012; 27: $331-336$
18 Jiang Z, Ge Z, Dai J et al. Comparison of autofluorescence imaging (AFI) and white light (WL) endoscopy in screening of colon polyps [in Chinese]. Chin J Dig Endosc 2010; 27: 173-178

19 Brown SR, Baraza W. Chromoscopy versus conventional endoscopy for the detection of polyps in the colon and rectum. Cochrane Database Syst Rev 2010; CD006439

20 Nagorni A, Bjelakovic G, Petrovic B. Narrow band imaging versus conventional white light colonoscopy for the detection of colorectal polyps. Cochrane Database Syst Rev; 2012: CD008361

21 Sabbagh LC, Reveiz L, Aponte D et al. Narrow-band imaging does not improve detection of colorectal polyps when compared to conventional colonoscopy: a randomized controlled trial and meta-analysis of published studies. BMC Gastroenterol 2011; 11: 100

22 Pasha SF, Leighton JA, Das A et al. Comparison of the yield and miss rate of narrow band imaging and white light endoscopy in patients undergoing screening or surveillance colonoscopy: a meta-analysis. Am J Gastroenterol 2012; 107: 363 - 370 quiz 371

23 Dinesen L, Chua TJ, Kaffes AJ. Meta-analysis of narrow-band imaging versus conventional colonoscopy for adenoma detection. Gastrointest Endosc 2012; 75: 604-611

24 van den Broek FJ, Reitsma JB, Curvers WL et al. Systematic review of narrow-band imaging for the detection and differentiation of neoplastic and nonneoplastic lesions in the colon (with videos). Gastrointest Endosc 2009; 69: 124-135

25 van Rijn JC, Reitsma JB, Stoker J et al. Polyp miss rate determined by tandem colonoscopy: a systematic review. Am J Gastroenterol 2006; 101 $343-350$

26 Pickhardt PJ, Choi JR, Hwang I et al. Computed tomographic virtual colonoscopy to screen for colorectal neoplasia in asymptomatic adults. $\mathrm{N}$ Engl J Med 2003; 349: 2191 - 2200

27 van den Broek FJ, van Soest EJ, Naber AH et al. Combining autofluorescence imaging and narrow-band imaging for the differentiation of adenomas from non-neoplastic colonic polyps among experienced and non-experienced endoscopists. Am J Gastroenterol 2009; 104 $1498-1507$ 